\title{
V25. PREVALENCE AND RISK FACTORS ASSOCIATED WITH HOOKWORM INFECTION IN NORTHEASTERN BRAZIL: ASSESSING THE POTENTIAL IMPACT OF A HUMAN HOOKWORM VACCINE.
}

Kerla Joeline Lima Monteiro ${ }^{1}$; Beatriz Coronato Nunes ${ }^{2}$; Lauren Hubert Jaeger ${ }^{3}$; Elis Regina Chaves dos Reis ${ }^{1}$; Jéssica Pereira Santos ${ }^{4}$; Deiviane Aparecida Calegar ${ }^{1}$; Filipe Anibal Carvalho-Costa ${ }^{1}$.

${ }^{1}$ Instituto Oswaldo Cruz / Fiocruz;

${ }^{2}$ Secretaria Municipal de Nossa Senhora de Nazaré, Piauí;

${ }^{3}$ Instituto Oswaldo Cruz / Fiocruz;

${ }^{4}$ Fiocruz, Piauí.

INTRODUCTION Among the soil-transmitted helminths, hookworms affect near 440 million people worldwide, motivating research for vaccine development. A vaccine composed with two Necator americanus recombinant antigens (glutathione-Stransferase $[\mathrm{Na}-\mathrm{GST}-1]$ and aspartic protease $[\mathrm{Na}-\mathrm{APR}-1])$, represented by enzymes involved in the hemoglobin uptake by hookworms has been passed in Phase 1 clinical trial in Brazil. Hookworm exposure to the host's blood (and hence to antibodies) make them susceptible to the action of vaccines.

OBJECTIVE This study aims to characterize prevalence and risk factors associated with hookworm infection in a rural area of Piauí state, northeastern Brazil, assessing the potential impact of a vaccine.

METHODOLOGY The study was carried out in the municipality of Nossa Senhora de Nazaré, Piauí state. A cross-sectional survey ( $\mathrm{n}=601$ subjects; 172 families/ houses) was carried out in 2014 and 2015. Maps were generated using the kernel density estimation method. Spatial data were analyzed in a GIS platform using ArcGis $9.3^{\circledR}$ software. Parasitological examinations were performed through the formalinethyl-acetate centrifugation and quantitative Kato-Katz smears.

RESULTS The overall prevalence of hookworm infection was 14\% (84/601). Prevalence rates in the rainy (2014) and dry (2015) seasons were $13.6 \%$ and $14.3 \%$, respectively $(\mathrm{p}=0.797)$, with higher positivity rate among subjects aged 13 to 21 years 
(18.6\%) and lower among persons aged > 60 years-old (9.3\%). Concerning families, the rate of detection of at least one positive subject was 39/172 (22.7\%). Positivity in families performing open defecation or using rudimentary tanks was significantly higher (31/107 [29\%]) than in houses with access to septic tanks (8/65 [12.3\%]), $\mathrm{p}=0.011$. Positivity in houses with uncoated clay floor was significantly higher than among families living in domiciles with ceramic-coated floor (11/27 [40.7\%] vs. 28/145 [19.3\%]; $\mathrm{p}=0.015)$. Among 84 hookworm-positive subjects, in 67 the infection intensity could be assessed: $62(92.5 \%)$ had low intensity, four (6\%) had moderate intensity and one (1.5\%) had heavy hookworm burden. Median parasitic load of infections was 168 eggs per gram (epg) of faeces, ranging from 24 to 4,368 epg. Intensity of hookworm infections presented a trend to increase inversely to age $(R=0.155 ; \mathrm{p}=0.213)$. Children aged 1-4 years presented the higher fecal egg counts (median=384 epg), while children aged 5-12 years presented median egg count $=120 \mathrm{epg}$, nevertheless differences were not statistically significant. Eggs had 57.2-80.2 $\mu \mathrm{m}$ in length, being compatible with hookworms, infection with the zoonotic Trichostrongylus sp. being discarded. One subject was infected with Trichuris trichiura (0.16\%). No infections with Ascaris lumbricoides were found. Geoprocessing identified hotspots with higher prevalence rates.

CONCLUSION Hookworm infection persists as a rural endemic disease with focal character, and some regions with specific demographic characteristics can produce high prevalence rates, with moderate parasitic loads. An effective vaccine would contribute to the control of hookworm in rural areas.

KEYWORDS hookworm, northeastern Brazil, prevelence, hookworm vaccine. 